\title{
A MEASURE THEORETIC VARIANT OF BLUMBERG'S THEOREM
}

\author{
JACK B. BROWN
}

\begin{abstract}
It is the purpose of this note to present a measure theoretic variant of Blumberg's theorem about continuous restrictions of arbitrary real functions.
\end{abstract}

Henry Blumberg [1] proved a theorem in 1922, which in its most basic form is stated as follows:

Theorem A. For every $f: I \rightarrow R$, there exists $D \subset I, D$ dense in $I$, such that $f \mid D$ is continuous.

$I$ is the interval [0, 1] and $R$ denotes the reals. Blumberg observed [2] that the set $D$ cannot be made to have cardinality $c$ because of the function $f$ : $I \rightarrow R$ of Sierpiński and Zygmund [6] which has no continuous restriction of cardinality $c$. However, the author established theorems [3] from which the following results:

THEOREM B. For every $f: I \rightarrow R$, there exists $W \subset I, W c$-dense in $I$, such that $f \mid W$ is pointwise discontinuous $(P W D)$.

$W$ is $c$-dense in $I$ if and only if every subinterval of I contains $c$-many points of $W$. A function $g$ with domain $W$ is PWD if and only if there exists $D \subset W, D$ dense in $W$, such that $g$ is continuous at each element of $D$.

P. Erdös recently asked the author if something could be done to make the set $W$ in the conclusion of Theorem B large relative to Lebesgue measure $\lambda$.

First, notice that it would be easy to alter the set $W$ in such a way to make it an $M$ set (i.e. have $\lambda^{0}(W)>0$ ). Let $D \subset W$ be the set on which $f \mid W$ is continuous, let $C$ be a Cantor subset of $I$ of positive measure, and let $W^{\prime}=W \cup C$. Then $\lambda^{0}\left(W^{\prime}\right)>0$, and $f \mid W^{\prime}$ is still continuous at each element of $D-(C \cap D)$, which is dense in $W^{\prime}$.

On the other hand, it would not be possible to make $W$ have outer measure 1 or even be $M$-dense in $I$ if $f$ is a function such as the following: let $C_{1}, C_{2}, \ldots$, be a sequence of disjoint Cantor subsets of $I$ such that $C_{1} \cup C_{2}$ $\cup \ldots$ has measure 1 , and let $f(x)=n$ if $x \in C_{n} . A$ is $M$-dense in $B$ if $A \subset B$ and every open set which intersects $B$ intersects $A$ in an $M$ set.

Next we might ask if $W$ can be made to be $M$-dense in itself and drop the

Received by the editors February 22, 1977.

AMS (MOS) subject classifications (1970). Primary 26A15.

( American Mathematical Society 1977 
requirement that it be dense in $I$. Dropping the requirement that $W$ be dense in $I$ will, in fact, make it possible to obtain differentiability on a dense subset of $W$ if infinite derivatives are allowed (i.e. $f$ is differentiable at $x$ if $f$ is continuous at $x, x$ is a limit point of $D_{f}$, and there is $t,-\infty \leqslant t \leqslant \infty$, such that if $\left\{x_{n}\right\}$ is a sequence of elements of $D_{f}-\{x\}$ converging to $x$, then $\left\{\left(f(x)-f\left(x_{n}\right)\right) /\left(x-x_{n}\right)\right\}$ converges to $\left.t\right)$. In [5] Ceder proved the following:

Theorem C. Suppose $X \subset I$ is uncountable. Then for every $f: X \rightarrow R$ there exists $D \subset X, D$ bilaterally dense in itself, such that $f \mid D$ is differentiable.

In [4] the author proved the following variant of Ceder's theorem:

TheOREM D. Suppose $X \subset I$ is not an $L_{1}$ set. Then for every $f: X \rightarrow R$ there exist $W \subset X$ and $D \subset W, W$ bilaterally $c$-dense in itself and $D$ dense in $W$ such that $f \mid W$ is differentiable at each element of $D$.

An $L_{1}$ set is a countable union $M_{1} \cup M_{2} \cup \ldots$ such that for each $i$, every nowhere dense in $M_{i}$ subset of $M_{i}$ has cardinality less than $c$. A set is bilaterally dense ( $c$-dense) ( $M$-dense) in itself if and only if every closed interval which intersects it intersects it in an infinite set (set of cardinality $c$ ) ( $M$ set). The converse of Theorem D was also shown to hold. An alteration of the proof of Theorem $\mathrm{D}$ will prove the following:

TheOREM E. Suppose $X \subset I$ is an $M$ set. Then for every $f: X \rightarrow R$, there exist $W \subset X$ and $D \subset W, W$ bilaterally $M$-dense in itself and $D$ dense in $W$, such that $f \mid W$ is differentiable at each element of $D$.

The proof of Theorem E can proceed almost identically with the proof of Theorem 1 of [4], with the notions " $L_{1}$ " and " $L_{2}$ " (which means "not $L_{1}$ ") of [4] replaced by "null" (which in this case means "of measure zero") and " $M$ ", respectively. Lemma 1 of [4] states that if $x$ is an element of a bilaterally $L_{2}$-dense in itself set $A$, then there exists a bilaterally $c$-dense in itself nowhere dense in $A$ subset $N$ of $A$ containing $x$. Indeed, the fact that the Continuum Hypothesis implies the existence of a Lusin set, so that the conclusion of Lemma 1 cannot be obtained if it is just known that $A$ is bilaterally $c$-dense in itself, is the cause of most of the difficulties encountered in proving Theorems $\mathrm{B}$ and $\mathrm{D}$, and brought about the necessity of defining properties $L_{1}$ and $L_{2}$. The situation with respect to $M$ sets is much simpler in the sense that Lemma 1 of [4] can be replaced by the following:

LEMMA $1^{\prime}$. If $x$ is an element of a bilaterally $M$-dense in itself set $A$, then there exists a bilaterally $M$-dense in itself nowhere dense in $A$ subset $N$ of $A$ containing $x$.

Proof. There exists a $G_{\delta}$ set $B$ such that $A \subset B$ and $\lambda^{0}(A)=\lambda(B)$. Assume that $B \subset \mathrm{Cl}(A)$, so that $B$ is $M$-dense in itself. It follows that if $C \subset B$ and $\lambda(C)>0$, then $\lambda^{0}(A \cap C)=\lambda(C)$. For each positive integer $n$, the set $A_{n}=[x, x+1 / n] \cap A$ has positive outer measure. Consider a Cantor set $C_{n}$ 
of positive measure such that $C_{n}$ is a subset of and nowhere dense relative to $[x, x+1 / n] \cap B$. Let $C_{n}{ }^{\prime}$ consist of the points of density 1 of $C_{n}$. Then $R_{n}=C_{n}{ }^{\prime} \cap A$ will be a bilaterally $M$-dense in itself subset of $[x, x+1 / n] \cap$ $A$ which is nowhere dense in $[x, x+1 / n] \cap A$. The set $[x-1 / n, x] \cap A$ will contain a similar set $L_{n}$. Then

$$
N=L_{1} \cup L_{2} \cup \ldots \cup(x) \cup R_{1} \cup R_{2} \cup \ldots
$$

is the desired set.

Now, Lemmas 2-6 of [4] can be altered by replacing " $L_{1}$ " and " $L_{2}$ " by "null" and " $M$ ", respectively, and the proofs will be the same. Then on stage (2) of the inductive procedure in the proof of Theorem $1\left[4\right.$, p. 39], make $N_{s}$ be bilaterally $M$-dense in itself, and Theorem $\mathrm{E}$ will be proved.

\section{REFERENCES}

1. H. Blumberg, New properties of all real functions, Trans. Amer. Math. Soc. 24 (1922), 113-128.

2. __ Arbitrary point transformations, Duke Math. J. 11 (1944), 671-685.

3. J. B. Brown, Metric spaces in which a strengthened form of Blumberg's theorem holds, Fund. Math. 71 (1971), 243-253.

4. Fund. Math. 84 (1974), 35-45.

5. J. G. Ceder, Differentiable roads for real functions, Fund. Math. 65 (1969), 351-358.

6. W. Sierpiński and A. Zygmund, Sur une function qui est discontinue sur tout ensemble de puissance du continu, Fund. Math. 4 (1923), 316-318.

Department of Mathematics, Auburn University, Auburn, Alabama 36830 